ISSN 0258-7122

Bangladesh J. Agril. Res. 36(1) : 173-181, March 2011

\title{
EFFECT OF WATER STRESS ON STOMATAL CHARACTERS OF TWENTY ONE NEAR ISOGENIC LINES OF WHEAT (Triticum aestivum L.)
}

\author{
A. M. S. ALAM ${ }^{1}$, G. KABIR ${ }^{2}$, M. M. UD-DEEN ${ }^{3}$ AND E. HOQUE ${ }^{4}$
}

\begin{abstract}
The present study was carried out to determine the effect of water stress on stomatal characters of flag leaf of wheat (Triticum aestivum L.) where five different irrigation regimes were considered as environments. Stomatal opening frequencies were significantly decreased by water stress in both the surfaces of the flag leaf in both irrigated and rainfed conditions in all the near isogenic lines of wheat. In rainfed condition, it was lower than irrigated condition. Similarly stomatal index also significantly differed in different irrigation treatments at three different positions in both adaxial and abaxial surface but under rainfed condition, it was lower than the irrigated condition in all the genotypes. The pore lengths of flag leaf in both adaxial and abaxial surfaces were found similar in size. It also varied among the different irrigations, but the variations were not remarkable. In rainfed condition, the pore lengths of different genotypes showed lower values than irrigated condition. Effects of water stress on stomatal pore breadths in both the surfaces were significantly decreased in rainfed condition. But in different irrigation conditions, stomatal pore breadth were non-significant among the genotypes. The effect of water stress on different stomatal characters in both surface of leaf in different lines of wheat were decreased.
\end{abstract}

Keywords: Water stress, stomatal characters, wheat .

\section{Introduction}

Rapid stomatal closure during the development of drought stress may account for the maintenance of higher water potentials in some cultivars. Stomatal aperture is an important index of drought tolerance since most transpirational water loss occurs through open stomata. Glover (1959) reported changes in stomatal response after repeated stress periods. Sullivan and Eastin (1975) pointed out the necessity of considering previous growth conditions when evaluating stomatal response to water stress. Several workers reported the importance of the structures above the flag-leaf node in contributing photosynthate to the developing grains of wheat (Carr and Wardlaw, 1965; Kriedemann, 1966; Quinlan and Sagar, 1965 and Voldeng and Simpson, 1967). Higher residual transpiration rates have been reported in leaves from irrigated than from rainfed field grown plants of wheat (Clark and McCaig, 1982). Senescence from the

\footnotetext{
${ }^{1 \& 2}$ Professor, Department of Botany, University of Rajshahi, ${ }^{3}$ Associate Professor, Department of Crop Science \& Technology, University of Rajshahi, ${ }^{4}$ Assistant Professor, Department of Botany, Godagari Degree College, Rajshahi, Bangladesh.
} 
lower to the upper leaves is accelerated (Asana et al., 1958) with the result that the total supply of photosynthate to the plant is reduced. Considering the above view points in minds, the present investigation was undertaken to determine the water stress sensivity for area and stomatal behaviour of flag leaves of twenty one Near Isogenic Lines (NILs) of wheat (Triticum aestivum L.) at various growth stages under different schedule of irrigations and rainfed condition.

\section{Materials and Method}

Twenty one Near Isogenic Lines (NILs) of wheat (Triticum aestivum L.) obtained from different crosses between four Bangladeshi varieties (Aghrani, Akbar. Ananda, and Kanchan) and two dwarf selected lines (FM-132 and FM-139) of wheat were used as plant materials. The seeds of these lines were procured from the Cytogenetics Laboratory, Department of Botany, University of Rajshahi, Bangladesh. The present investigations were conducted in the experimentation field of Rajshahi University. The experimentation fields were prepared after repeated ploughings. The soil was measured properly. Uniform and saturated intercultural operations were done as and when necessary for all traits to raise the better crop. The Near Isogenic Lines of wheat were raised in split plot with three replications and evaluated at five irrigation (I) conditions: $I_{3}$ (irrigation was given at the root crowning stage, $\mathrm{I}_{2}\left(\mathrm{I}_{2}\right.$, + at the tillering stage $), \mathrm{I}_{3}\left(\mathrm{I}_{3}+\right.$ at the booting stage), $\mathrm{I}_{3}\left(\mathrm{I}_{3}+\right.$ at the heading stage), and $\mathrm{I}_{0}$ (rainfed condition) treated as control. Transparent quick fix adhesives were spread on both the adaxial and abaxial surfaces of flag leaf. Allowed to dry for 30 minutes and then thin film of quick fix were taken out with the help of a fine forceps. The numbers of stomata from 30 random focuses from each surface of leaves were counted under a compound microscope and subsequently, the values were converted and expressed as number per $\mathrm{mm}^{2}$ of leaf area.

The pore length and pore breadth of stomata of leaves were measured with the help of occulometer in a compound microscope using the values were converted into micron $(\mu \mathrm{m})$ and the stomatal index of both ahaxial and adaxial surfaces of flag leaf of different genotypes were calculated and the data were analyzed statistically using conventional method.

\section{Results and Discussion}

Stomata are apparatus in the epidermis each bounded by two guard cells. In green leaves of monocotyledons, these stomata are arranged in parallel rows. In the present study, significant effect of soil moisture on stomatal number or density of stomata per $\mathrm{mm}^{2}$ on both the adaxial and abaxial surfaces was found (Table 1). The stomatal number on the adaxial surface was found to be more than on the abaxial surface. Similar results were reported by Kazemi et al., (1978) and Volkenbirrgh and Davies (1977). Number of stomata in both in adaxial and 
Table 1. Mean values of stomata number per $\mathbf{m m}^{2}$ of both adaxial and abaxial surface of leaf in twentyone near isogenic lines along with a check variety kanchan of wheat (Triticum aestivum $\mathrm{L}_{\text {.) }}$ in different irrigation (I) condition.

\begin{tabular}{|c|c|c|c|c|c|c|c|c|c|c|}
\hline \multirow{3}{*}{$\begin{array}{c}\text { Genotypes } \\
\text { (G) }\end{array}$} & \multicolumn{5}{|c|}{ Adaxial surface } & \multicolumn{5}{|c|}{ Abaxial surface } \\
\hline & \multicolumn{5}{|c|}{ Irrigation (I) } & \multicolumn{5}{|c|}{ Irrigation (I) } \\
\hline & Control & $\mathrm{I}_{1}$ & $\mathrm{I}_{2}$ & $\mathrm{I}_{3}$ & $\mathrm{I}_{4}$ & Control & $\mathrm{I}_{1}$ & $\mathrm{I}_{2}$ & $\mathrm{I}_{3}$ & $\mathrm{I}_{4}$ \\
\hline G1 & 40.33abc & $44.33 \mathrm{~b}-\mathrm{f}$ & 43.67de & 43.67bcd & 42.00efg & 33.33bcd & $38.00 \mathrm{~cd}$ & $39.67 \mathrm{abc}$ & $39.33 \mathrm{bc}$ & $38.67 b$ \\
\hline G2 & 40.67ab & 45.00b-e & 43.33def & $44.67 b$ & 44.33bcd & $34.67 b$ & 39.00bc & 37.33def & 37.33de & $37.67 \mathrm{bc}$ \\
\hline G3 & 39.33a-d & 43.33d-g & 44.67cde & 43.67bcd & $45.00 \mathrm{~b}$ & 37.33a & $41.67 \mathrm{a}$ & 40.33ab & $41.67 \mathrm{a}$ & $42.00 \mathrm{a}$ \\
\hline G4 & $38.67 \mathrm{~b}-\mathrm{e}$ & 42.33fgh & 41.00g-j & 43,67bcd & 41.33fg & $34.67 b$ & $40.67 \mathrm{ab}$ & 36.67efg & 36.67def & 38.33bc \\
\hline G5 & $38.33 c-f$ & 43.00efg & 44.33de & 43.67bcd & 43.67b-e & 31.33efg & 35.67efg & 35.00ghi & 36.33efg & 34.33efg \\
\hline G6 & $41.00 \mathrm{a}$ & 47.33a & $45.67 \mathrm{bcd}$ & $44.00 \mathrm{bc}$ & $44.00 \mathrm{~b}-\mathrm{e}$ & $30.67 f g$ & $34.67 \mathrm{fgh}$ & 35.00ghi & $34.33 \mathrm{~h}-\mathrm{k}$ & 34.67ef \\
\hline G7 & $38.33 c-f$ & 40.67hi & 43.00efg & $41.67 d-g$ & $42.67 c-f$ & $28.33 i$ & $32.00 \mathrm{j}$ & $33.00 \mathrm{jk}$ & $32.67 \mathrm{k} 1$ & $32.33 \mathrm{~h}$ \\
\hline G8 & 37.33d-f & 41.67gh & 42.67e-h & 40.67fgh & 41.00fgh & 30.33gh & $34.00 \mathrm{f}-\mathrm{i}$ & $33.67 \mathrm{ijk}$ & $34.33 \mathrm{~h}-\mathrm{k}$ & 33.67fgh \\
\hline G9 & $36,33 f$ & 39.33ij & 4067hij & $39.00 \mathrm{~h}$ & 41.33fg & 32.33def & $36.00 \mathrm{ef}$ & 34.67hij & $36.00 \mathrm{e}-\mathrm{h}$ & 35.00def \\
\hline G10 & 40.00abc & 44.33b-f & 46.67abc & $42.33 c-f$ & $45.00 \mathrm{~b}$ & 30.33gh & 34.67fgh & 34.33h-k & 41.00ab & 36.67cd \\
\hline G11 & 36.67ef & 37.33j & 41.33f-i & $39.00 \mathrm{~h}$ & 40.00gh & 32.67cde & 36.67de & 34.33h-k & $35.00 \mathrm{f}-\mathrm{j}$ & 35.67de \\
\hline G12 & 37.67def & 41.33ghi & $39.00 \mathrm{j}$ & 41.33efg & $39.00 \mathrm{~h}$ & 30.00ghi & $34.00 f-i$ & 35.00ghi & $34.33 \mathrm{~h}-\mathrm{k}$ & 35.00def \\
\hline G13 & $40.00 \mathrm{abc}$ & $44.00 \mathrm{c}-\mathrm{f}$ & 48.33a & 47.33a & $48.00 \mathrm{a}$ & $30.67 f g$ & $34.67 \mathrm{fgh}$ & $35.67 \mathrm{fgh}$ & $39.67 b c$ & $38.67 \mathrm{~b}$ \\
\hline G14 & 37.33def & 43.00efg & 43.67de & $42.00 \mathrm{c}-\mathrm{f}$ & $44.67 \mathrm{bc}$ & 33.33bcd & 38.33cd & 38.67bcd & 38.33cd & 38.33bc \\
\hline G15 & 37.33def & 42.67fgh & $39.33 \mathrm{ij}$ & 39.67gh & 40.00gh & $34.33 b c$ & 38.33cd & 38.00cde & 38.33cd & 38.33bc \\
\hline G16 & 38.67b-e & $44.00 \mathrm{c}-\mathrm{f}$ & 42.67e-h & $44.67 \mathrm{~b}$ & $44.00 \mathrm{~b}-\mathrm{e}$ & 28.67hi & 32.33ij & 33.67ijk & 34.67g-j & 34.67ef \\
\hline G17 & 40.67ab & $46.00 \mathrm{abc}$ & $47.00 \mathrm{ab}$ & $44.00 \mathrm{bc}$ & $46.00 \mathrm{ab}$ & $30.67 \mathrm{fg}$ & 34.33fgh & 34.33h-k & $36.00 \mathrm{e}-\mathrm{h}$ & 34 33efg \\
\hline G18 & 38.33c-f & 45.33a-d & 44.00de & $44.67 \mathrm{~b}$ & $45.67 b$ & 30.33gh & 33.67g-j & $32.67 \mathrm{k}$ & 32.331 & 32.67gh \\
\hline G19 & $41.00 \mathrm{a}$ & 46.33ab & 46.67abc & 45.33ab & 44.67bc & 31.33efg & 35.33efg & 34.67fgh & $35.67 \mathrm{e}-\mathrm{i}$ & 35.00def \\
\hline G20 & 37.33def & 44.33b-f & 44.00de & $44.67 \mathrm{~b}$ & 42.33dcfr & $30.67 \mathrm{fg}$ & 35.00efg & 34.67hij & 33.67jk1 & 36.67cd \\
\hline G21 & 37.33dcf & 40.67hi & $40.00 \mathrm{ij}$ & 43.33b-e & 42.67c-f & 28.67hi & 33.00hij & $32.67 \mathrm{k}$ & 34.00i-l & 34.67ef \\
\hline Kanchan & 39.33a-d & 45.33a-d & $45.00 \mathrm{~b}-\mathrm{e}$ & 43.67bcd & $44.00 \mathrm{~b}-\mathrm{e}$ & 33.33bcd & 39.33bc & 41.33a & $42.00 \mathrm{a}$ & 41.33a \\
\hline $\mathrm{CV}(\%)$ & & & 3.0 & & & & & 3.1 & & \\
\hline
\end{tabular}

In a column, means followed by a common letter are not significantly different at the 5\% 1ee1 by DMRT. 
Table 2. Mean values of stomatal index (\%) of both adaxial and abaxial surface of leaf in twenty one near isogenic lines along with a check variety kanchan of wheat (Triticum aestivum $\mathrm{L}$.) in different irrigations (I).

\begin{tabular}{|c|c|c|c|c|c|c|c|c|c|c|}
\hline \multirow{3}{*}{$\begin{array}{c}\text { Genotypes } \\
\text { (G) }\end{array}$} & \multicolumn{5}{|c|}{ Adaxial surface } & \multicolumn{5}{|c|}{ Abaxial surface } \\
\hline & \multicolumn{5}{|c|}{ Irrigation (I) } & \multicolumn{5}{|c|}{ Irrigation (I) } \\
\hline & Control & $\mathrm{I}_{1}$ & $\mathrm{I}_{2}$ & $\mathrm{I}_{3}$ & $\mathrm{I}_{4}$ & Control & $\mathrm{I}_{1}$ & $\mathrm{I}_{2}$ & $\mathrm{I}_{3}$ & $\mathrm{I}_{4}$ \\
\hline G1 & 67.02cde & $67.35 f g$ & 68.57c-f & $68.40 \mathrm{e}-\mathrm{h}$ & $66.32 \mathrm{~g}$ & $63.74 c$ & $66.06 c$ & $66.06 c$ & $66.65 b$ & 66.8lab \\
\hline G2 & $69.02 \mathrm{a}$ & $70.41 \mathrm{a}$ & 70.03a & 70.06 & $70.14 a$ & 64.37b & $67.67 \mathrm{a}$ & $67.67 a$ & 66.73ab & 66.79ab \\
\hline G3 & 66.28d-h & 66.08hi & $66.42 \mathrm{~g}$ & 66.66jk & 68.00def & 64.98a & 66.91b & 66.91b & $66.25 b c$ & $67.22 \mathrm{a}$ \\
\hline G4 & 63.30k & 68.17c-f & $67.72 \mathrm{f}$ & 69.33b-e & 67.84def & 64.70ab & 67.42ab & 67.42ab & 65.llef & 66.68ab \\
\hline G5 & $66.22 \mathrm{~d}-\mathrm{i}$ & 68.7lbcd & 68.93bcd & 69.42bcd & 69.12bc & 62.39d & 64.85de & 64.85de & $65.84 \mathrm{~cd}$ & $65.25 \mathrm{ef}$ \\
\hline G6 & $65.80 f-i$ & 67.84def & $67.30 \mathrm{~g}$ & 65.751 & $66.02 \mathrm{~g}$ & $60.43 f$ & 61.94hi & 61.94hi & $62.80 \mathrm{i}$ & $62.70 \mathrm{k}$ \\
\hline G7 & $68.00 \mathrm{~b}$ & $69.24 b$ & 68.87bcd & $69.04 c-f$ & 69.1lbc & $58.58 \mathrm{~h}$ & $60.79 \mathrm{j}$ & $60.79 \mathrm{j}$ & $61.88 \mathrm{j}$ & 61.711 \\
\hline G8 & 67.llcd & 68.50b-e & 68.74b-e & $70.35 a$ & 67.65ef & 60.95ef & 63.61g & $63.61 \mathrm{~g}$ & $63.62 \mathrm{~h}$ & 63.76ij \\
\hline G9 & 66.23d-i & $64.86 j$ & 68.94bcd & 68.0lgh & $69.05 c$ & 62.84d & 64.55ef & $64.55 \mathrm{ef}$ & 65.44de & 64.27ghi \\
\hline G10 & 65.62f-i & $67.32 f g$ & 67.80efg & 65.83k1 & $66.18 \mathrm{~g}$ & $59.52 \mathrm{~g}$ & 62.04hi & 62.04hi & 65.54de & 64.57gh \\
\hline G11 & $62.77 \mathrm{k}$ & $65.34 \mathrm{ij}$ & $67.67 \mathrm{fg}$ & 67.09ij & 67.57ef & $52.19 \mathrm{i}$ & 66.21c & $66.21 c$ & 65.45de & 65.37de \\
\hline G12 & 65.33hi & $66.45 \mathrm{gh}$ & $65.59 \mathrm{~h}$ & 67.63hi & 65.88gh & $62.46 \mathrm{~d}$ & 64.65ef & $64.65 \mathrm{ef}$ & 64.78fg & 64.78fg \\
\hline G13 & 66.57c-f & 68.21c-f & 70.03a & 69.10c-f & 70.04ab & 62.63d & 64.69e & 64.69e & $66.76 \mathrm{ab}$ & 66.39bc \\
\hline G14 & $64.36 \mathrm{j}$ & $67.24 f g$ & 67.89efg & 67.0lij & 68.49cde & $62.54 d$ & $65.36 \mathrm{~d}$ & $65.36 \mathrm{~d}$ & $64.43 \mathrm{~g}$ & 65.40ge \\
\hline G15 & 66.82cde & 66.13hi & $64.58 \mathrm{i}$ & $64.89 \mathrm{~m}$ & $65.12 \mathrm{~h}$ & 64.2lbc & $65.95 c$ & $65.95 c$ & $66.40 \mathrm{bc}$ & 65.89cd \\
\hline G16 & 66.41dg & $69.26 \mathrm{~b}$ & 68.40def & 69.85abc & 69.0lc & $61.55 \mathrm{e}$ & 63.72g & $63.72 \mathrm{~g}$ & $64.49 \mathrm{~g}$ & 65.84cde \\
\hline G17 & 67.39bc & $69.29 \mathrm{~b}$ & $70.16 a$ & 68.22fgh & 69.28abc & $61.14 \mathrm{e}$ & $64.05 f g$ & $64.05 f g$ & 64.59fg & $63.31 \mathrm{j}$ \\
\hline G18 & 65.50ghi & $68.64 b-c$ & 69.50abc & 68.26fgh & 69.36abc & $60.43 f$ & $62.53 \mathrm{~h}$ & $62.53 \mathrm{~h}$ & $60.66 \mathrm{k}$ & 61.851 \\
\hline G19 & 65.81f-i & 67.67ef & 69.32a-d & $68.70 \mathrm{~d}-\mathrm{g}$ & 67.64ef & $62.42 d$ & 64.43ef & 64.43ef & $64.75 f g$ & 64.34ghi \\
\hline G20 & $65.23 \mathrm{i}$ & 68.52b-e & 68.48def & 68.llfgh & $67.44 \mathrm{f}$ & 61.53e & 64.04fg & 64.06fg & $63.42 \mathrm{~h}$ & 65.48de \\
\hline G21 & $65.70 f-I$ & $67.53 f$ & $67.37 \mathrm{~g}$ & $68.84 d-g$ & 68.47cde & $59.50 \mathrm{~g}$ & 61.651 & 61.651 & $63.79 \mathrm{~h}$ & 64.03hi \\
\hline Kanchan & 66.08e-i & 68.84bc & 69.58ab & 68.87d-g & 68.74cd & $62.72 d$ & $63.78 \mathrm{~g}$ & $63.78 \mathrm{~g}$ & $67.26 \mathrm{a}$ & 66.83ab \\
\hline
\end{tabular}

In a column, means followed by a common letter are not sinific-ant1y different at the $5 \%$ level by DMRT. 
Table 3. Mean values of pore length $(\mu \mathrm{m})$ of both adaxial and abaxial surface of leaf in twenty one near isogenic lines along with a check variety Kanchan of wheat (Triticum aestivum $\mathrm{L}$.) in different irrigations (I)

\begin{tabular}{|c|c|c|c|c|c|c|c|c|c|c|}
\hline \multirow{3}{*}{ Genotypes (G) } & \multirow{2}{*}{\multicolumn{5}{|c|}{$\begin{array}{c}\text { Adaxial surface } \\
\text { Irrigation (I) }\end{array}$}} & \multirow{2}{*}{\multicolumn{5}{|c|}{$\begin{array}{c}\text { Abaxial surface } \\
\text { Irrigation (I) }\end{array}$}} \\
\hline & & & & & & & & & & \\
\hline & Control & $\mathrm{I}_{1}$ & $\mathrm{I}_{2}$ & $\mathrm{I}_{3}$ & $\mathrm{I}_{4}$ & Control & $\mathrm{I}_{1}$ & $\mathrm{I}_{2}$ & $\mathrm{I}_{3}$ & $\mathrm{I}_{4}$ \\
\hline G1 & $5.63 \mathrm{~b}-\mathrm{f}$ & $5.84 \mathrm{bc}$ & 5.95cde & $5.86 \mathrm{bcd}$ & $5.62 \mathrm{c}-\mathrm{f}$ & $6.16 \mathrm{~d}$ & 6.48cde & 6.49cde & $6.36 c-f$ & 6.34de \\
\hline G2 & $5.71 \mathrm{bf}$ & $6.33 b$ & 6.03b-e & $6.22 \mathrm{bc}$ & 6.06a-e & $6.24 \mathrm{~cd}$ & 6.52 cde & 6.21d-h & $6.31 c-f$ & 6.48bcd \\
\hline G3 & 5.46def & $6.03 \mathrm{bc}$ & 5.92cde & 5.77cde & 5.95b-e & $7.20 \mathrm{a}$ & $7.45 a$ & 7.41a & 741a & 7.34a \\
\hline G4 & 5.50c-f & $6.24 \mathrm{~b}$ & 6.07b-e & $6.27 \mathrm{bc}$ & 6.lla-d & 5.79de & 6.69efg & $6.42 \mathrm{e}-\mathrm{g}$ & $6.58 \mathrm{bcd}$ & $6.54 \mathrm{bcd}$ \\
\hline G5 & $5.78 b-f$ & $6.08 \mathrm{bc}$ & 6.04b-e & $6.22 b c$ & 6.05b-e & $6.24 \mathrm{~cd}$ & 6.69bcd & 6.63bcd & $6.30 c-f$ & 6.44b-e \\
\hline G6 & 5.85b-e & $6.34 b$ & 6.16a-d & $6.08 \mathrm{bc}$ & 6.23ab & $6.09 \mathrm{~d}$ & 6.35d-g & $6.47 c-f$ & 6.59bcd & 6.39cde \\
\hline G7 & 5.68b-f & $5.82 \mathrm{bc}$ & 6.02b-e & $6.10 \mathrm{bc}$ & 6.18abc & $6.75 \mathrm{ab}$ & $6.92 \mathrm{bc}$ & 6.98ab & $6.89 \mathrm{~b}$ & 6.87abc \\
\hline G8 & 5.85b-e & $6.08 \mathrm{bc}$ & 6.02b-e & 6.04bcd & 6.14abc & $6.08 \mathrm{~d}$ & $6.37 d-g$ & 6.53bcd & 6.5lbcd & 6.36de \\
\hline G9 & $6.08 \mathrm{bc}$ & $6.00 \mathrm{bc}$ & 6.06b-e & 5.85bcd & 593b-e & $6.07 d$ & $6.26 \mathrm{~d}-\mathrm{g}$ & 6.14d-h & 6.16d-g & 6.12def \\
\hline G10 & 5.79b-f & $5.95 \mathrm{bc}$ & 5.6ldef & $6.09 \mathrm{bc}$ & 6.17abc & $6.20 \mathrm{~d}$ & $6.32 \mathrm{~d}-\mathrm{g}$ & 6.39c-h & 642bcd & 6.03def \\
\hline G11 & 5.99bcd & $6.20 \mathrm{~b}$ & 5.92cde & 5.7lcde & 6.13abc & $6.20 \mathrm{~d}$ & $6.27 \mathrm{~d}-\mathrm{g}$ & $6.45 c-g$ & $6.34 c-f$ & 6.40cde \\
\hline G12 & $5.50 c-f$ & $5.59 c$ & $5.36 f$ & $6.02 \mathrm{bcd}$ & 6.17abc & $6.05 d$ & $6.24 \mathrm{~d}-\mathrm{g}$ & 6.38c-h & $6.08 \mathrm{~d}-\mathrm{g}$ & 6.30de \\
\hline G13 & 5.65b-f & $6.21 b$ & 6.33abc & $6.08 b c$ & 6.19abc & $6.24 \mathrm{~cd}$ & $6.27 \mathrm{~d}-\mathrm{g}$ & 5.96fgh & 6.lld-g & 6.37cde \\
\hline G14 & 5.94b-e & $6.03 \mathrm{bc}$ & 5.98cde & 6.99a & 6.13abc & $6.16 \mathrm{~d}$ & 6.33d-g & $6.24 c-h$ & 6.37cde & 6.4lcde \\
\hline G15 & $6.74 a$ & 7.lla & $6.64 a$ & 5.49de & $6.64 a$ & $6.28 \mathrm{~cd}$ & $6.30 \mathrm{~d}-\mathrm{g}$ & $6.23 \mathrm{~d}-\mathrm{h}$ & $6.34 c-f$ & 6.27def \\
\hline G16 & $5.36 \mathrm{ef}$ & $5.60 c$ & 5.62def & $6.07 \mathrm{bc}$ & 5.52ef & 5.76de & $5.93 f g$ & 6.15d-h & $5.67 \mathrm{~g}$ & 5.92efg \\
\hline G17 & 5.64b-f & $5.98 b c$ & 6.15a-e & $6.40 \mathrm{bc}$ & 5.85b-f & $6.70 \mathrm{bc}$ & $7.04 \mathrm{ab}$ & 7.00ab & $6.69 b c$ & 6.9lab \\
\hline G18 & 6.llb & $6.24 b$ & 6.58ab & $5.27 \mathrm{e}$ & 5.86b-f & $5.54 \mathrm{c}$ & $5.86 \mathrm{~g}$ & 5.97e-h & 5.88efg & $5.55 \mathrm{~g}$ \\
\hline G19 & $5.28 f$ & $5.57 c$ & $5.55 \mathrm{ef}$ & 6.19bc & $5.34 \mathrm{f}$ & 6.88ab & 7.03ab & $6.75 b c$ & $6.89 \mathrm{~b}$ & $7.22 \mathrm{a}$ \\
\hline G20 & 5.88b-e & $6.18 \mathrm{bc}$ & 6.12a-e & $6.05 \mathrm{bcd}$ & 6.09a-e & 5.85de & 6.00efg & $5.89 \mathrm{~h}$ & $5.86 f g$ & $5.80 \mathrm{fg}$ \\
\hline G21 & 5.58b-f & $5.8 \mathrm{lbc}$ & $5.84 c-f$ & $6.39 b$ & 5.55def & 5.87de & 6.0lefg & 5.93gh & $5.78 \mathrm{~g}$ & 6.36de \\
\hline Kanchan & 5.69b.f & $6.0 \mathrm{lbc}$ & 6.10a-e & $6.20 \mathrm{bc}$ & $6.40 \mathrm{ab}$ & $6.19 \mathrm{~d}$ & 6.39def & 6.35c-h & 6.31c-f & 6.37cde \\
\hline CV(\%) & & & 5.1 & & & & & 4.2 & & \\
\hline
\end{tabular}

In a column, means followed by a common letter are not significantly different at the $5 \%$ level by DMRT. 
Table 4. Mean values of pore breadth $(\mu \mathrm{m})$ of both adaxial and abaxial surface of leaf in twenty one near isogenic lines along with a check variety Kanchan of wheat (Triticum aestivum $\mathrm{L}$ ) in different irrigations (I).

\begin{tabular}{|c|c|c|c|c|c|c|c|c|c|c|}
\hline \multirow{3}{*}{ Genotypes (G) } & \multicolumn{5}{|c|}{ Adaxial surface } & \multicolumn{5}{|c|}{ Abaxial surface } \\
\hline & \multicolumn{5}{|c|}{ Irrigation (I) } & \multicolumn{5}{|c|}{ Irrigation (I) } \\
\hline & Control & $\mathrm{I}_{1}$ & $\mathrm{I}_{2}$ & $\mathrm{I}_{3}$ & $\mathrm{I}_{4}$ & Control & $\mathrm{I}_{1}$ & $\mathrm{I}_{2}$ & $\mathrm{I}_{3}$ & $\mathrm{I}_{4}$ \\
\hline G1 & $0.61 \mathrm{~g}-\mathrm{k}$ & 0.64fgh & 0.73ghi & $0.63 g$ & $0.60 \mathrm{j}$ & 0.66 cde & $0.64 c-f$ & 0.67 fgh & 0.64gh & 0.68def \\
\hline G2 & $0.61 \mathrm{~g}-\mathrm{k}$ & 0.64fgh & $0.7 \mathrm{lhi}$ & $0.65 g$ & 0.6lij & $0.60 \mathrm{ef}$ & 0.6lef & $0.70 \mathrm{e}-\mathrm{h}$ & $0.57 \mathrm{~h}$ & $0.62 \mathrm{f}$ \\
\hline G3 & $0.63 \mathrm{e}-\mathrm{i}$ & $0.76 \mathrm{~cd}$ & $0.87 \mathrm{~cd}$ & 0.76 def & $0.74 \mathrm{efg}$ & 0.63def & $0.71 a-e$ & $0.82 \mathrm{~b}-\mathrm{e}$ & 0.7lefg & $0.79 \mathrm{~cd}$ \\
\hline G4 & $0.88 \mathrm{~b}$ & $0.85 b$ & $0.95 \mathrm{ab}$ & $0.79 c-f$ & $0.85 b c$ & $0.72 \mathrm{bcd}$ & $0.76 \mathrm{abc}$ & $0.66 \mathrm{gh}$ & $1.00 \mathrm{ab}$ & $0.74 \mathrm{c}-\mathrm{f}$ \\
\hline G5 & $0.56 \mathrm{j}-\mathrm{m}$ & $0.61 \mathrm{~h}$ & 0.80d-g & $1.03 \mathrm{ab}$ & $0.68 \mathrm{ghi}$ & 0.6ldef & 0.62 def & 0.81b-e & $0.9 \mathrm{lbc}$ & 0.76 cde \\
\hline G6 & $0.55 \mathrm{k} 1 \mathrm{~m}$ & $0.7 \mathrm{lc}-\mathrm{f}$ & $0.90 \mathrm{bc}$ & $1.09 \mathrm{a}$ & $0.7 \mathrm{lfgh}$ & $0.59 \mathrm{ef}$ & $0.74 a-d$ & $0.76 \mathrm{~d}-\mathrm{g}$ & $1.02 \mathrm{a}$ & 0.68def \\
\hline G7 & $0.59 \mathrm{i}-\mathrm{l}$ & $0.66 \mathrm{e}-\mathrm{h}$ & $0.82 \mathrm{c}-\mathrm{f}$ & $0.76 \mathrm{c}-\mathrm{f}$ & $0.77 \mathrm{def}$ & 0.61def & 0.69a-e & $0.81 b-e$ & $0.81 \mathrm{c}-\mathrm{f}$ & 0.68def \\
\hline G8 & $0.54 \mathrm{klm}$ & 0.63gh & 0.85 cde & $0.83 \mathrm{~cd}$ & $0.63 \mathrm{ij}$ & $0.57 \mathrm{ef}$ & $0.64 \mathrm{c}-\mathrm{f}$ & $1.00 \mathrm{a}$ & $0.74 d-g$ & $0.65 \mathrm{df}$ \\
\hline G9 & $0.55 \mathrm{k} 1 \mathrm{~m}$ & 0.63gh & 0.79efg & $0.79 c-f$ & $0.80 \mathrm{~b}-\mathrm{e}$ & $0.57 \mathrm{ef}$ & 0.62def & 0.81b-e & 0.78def & $0.82 \mathrm{c}$ \\
\hline G10 & $0.61 \mathrm{~g}-\mathrm{k}$ & $0.69 d-g$ & 0.82def & $0.78 \mathrm{c}-\mathrm{f}$ & $0.64 \mathrm{hij}$ & $0.78 \mathrm{~b}$ & $0.68 b-f$ & $0.82 \mathrm{bcd}$ & $0.85 \mathrm{~cd}$ & $0.73 \mathrm{c}-\mathrm{f}$ \\
\hline G11 & 0.70 cde & 0.72cde & $0.84 c-f$ & 1.03ab & $0.67 \mathrm{ghi}$ & $0.98 \mathrm{a}$ & 0.70a-e & $0.86 \mathrm{bcd}$ & $0.69 f g$ & 0.68def \\
\hline G12 & $0.531 \mathrm{~m}$ & $0.60 \mathrm{~h}$ & $0.77 \mathrm{fgh}$ & $0.84 \mathrm{c}$ & $0.74 \mathrm{efg}$ & $0.55 \mathrm{ef}$ & $0.57 f$ & $0.82 \mathrm{~b}-\mathrm{e}$ & 1.0lab & 0.68def \\
\hline G13 & $0.511 \mathrm{~m}$ & 0.67e-h & $0.77 f g h$ & 0.8lcde & 0.66hij & $0.52 \mathrm{f}$ & $0.64 c-f$ & $0.79 c-f$ & 0.82cde & $0.96 \mathrm{a}$ \\
\hline G14 & $0.76 \mathrm{c}$ & $0.76 \mathrm{~cd}$ & 0.86cde & 0.82cde & 0.85bcd & $0.76 \mathrm{bc}$ & 0.79ab & $0.90 \mathrm{abc}$ & $0.75 d-g$ & 0.93ab \\
\hline G15 & $0.73 \mathrm{~cd}$ & 0.62gh & 0.8ldef & $1.00 \mathrm{~b}$ & $0.97 a$ & $0.78 \mathrm{~b}$ & $0.69 a-f$ & $0.81 \mathrm{~b}-\mathrm{e}$ & 0.70 efg & $0.95 \mathrm{a}$ \\
\hline G16 & $0.63 f-j$ & 0.64fgh & $0.80 \mathrm{~d}-\mathrm{g}$ & $0.75 \mathrm{ef}$ & $0.74 \mathrm{efg}$ & $0.59 \mathrm{ef}$ & $0.64 c-f$ & $0.76 \mathrm{~d}-\mathrm{g}$ & 0.72efg & 0.76cde \\
\hline G17 & $0.64 \mathrm{e}-\mathrm{i}$ & $0.76 \mathrm{~cd}$ & $0.77 \mathrm{fgh}$ & $0.78 \mathrm{c}-\mathrm{f}$ & $0.98 a$ & 0.65cde & $0.74 a-d$ & $0.77 d-g$ & $0.75 \mathrm{~d}-\mathrm{g}$ & $1.02 \mathrm{a}$ \\
\hline G18 & $1.00 \mathrm{a}$ & $1.15 a$ & $0.68 \mathrm{i}$ & $0.98 b$ & $0.53 \mathrm{k}$ & $0.75 b c$ & 0.69a-f & 0.80b-e & 0.72efg & 0.69def \\
\hline G19 & 0.67d-h & $0.86 b$ & 1.0la & $0.79 c-f$ & $0.78 \mathrm{c}-\mathrm{f}$ & 0.66cde & $0.81 \mathrm{a}$ & 0.9labc & $0.68 f g$ & $0.84 \mathrm{bc}$ \\
\hline G20 & $0.61 \mathrm{~h}-\mathrm{k}$ & $0.76 \mathrm{~cd}$ & 0.86cde & 0.8 lcde & $0.8 \mathrm{lb}-\mathrm{e}$ & $0.60 \mathrm{def}$ & 0.73a-e & $0.85 b c d$ & 0.82 cde & $0.82 \mathrm{c}$ \\
\hline G21 & $0.68 \mathrm{~d}-\mathrm{g}$ & 0.72cde & $0.83 c-f$ & $0.73 \mathrm{f}$ & $0.86 b$ & $0.64 c-f$ & 0.73a-e & $0.64 \mathrm{~h}$ & $0.74 d-g$ & $0.95 a$ \\
\hline Kanchan & $0.70 \mathrm{c}-\mathrm{f}$ & $0.77 \mathrm{c}$ & 0.56 cde & 0.5lcde & $0.77 \mathrm{ef}$ & 0.65 cde & $0.75 \mathrm{abc}$ & $0.92 \mathrm{ab}$ & $0.74 \mathrm{~d}-\mathrm{g}$ & 0.77 cde \\
\hline $\mathrm{CV}(\%)$ & & & 5.3 & & & & & 8.7 & & \\
\hline
\end{tabular}

In a column, means followed by a common letter are not significantly different at the $5 \%$ level by DMRT. 
abaxial surface of the flag leaf showed no such significant difference in different genotypes of wheat grown at irrigated and rainfed conditions. The present results also agreed with the findings reported by Jones (1977). It was also found that stomatal frequency per unit area varied among twenty one Near Isogenic Lines and check variety Kanchan (Table 1). Similar results were reported by Kazemi et al., 1978 and Naveem and Garskin, 1990 and they reported that the number of stomata per unit leaf area has been shown to vary among genotypes within species and to be under genetic control. In the present study, the results of stomatal frequency of flag leaf in both the surfaces revealed that the adaxial surface contained more stomata than that of abaxial surface. Similar results were also reported by Kumer et al. (1986) in wheat. In the present study, stomatal index was found to be decreased in rainfed condition and differed in different schedules of irrigation but not remarkable and not in any particular direction. It was also indicated that stomatal index were higher at upper surface than the lower (Table 2). These results are consistent with Volkenburgh and Davies (1977). The present fmdings indicated that the water stress was significantly influenced on stomatal index.

The mature stomatal apparatus consists of a lenticular pore with fine cuticular ledges and is surrounded by two kidney shaped guard - cells containing abundant chloroplast. Stomatal aperture is an important index of drought tolerance since most transpirational water loss occurs through open stomata. In addition with different physiological factors, among the environmental factor $\mathrm{CO}_{2}$ concentration appear to play a major role inside changes of the stomatal pore. Stomatal pore length of different genotypes at different schedules of irrigation in both the surfaces of flag leaves were found to vary but the differences were not remarkable and not in any particular direction. On the other hand, it was observed that stomatal pore lengths were significantly decreased in both the surfaces at rainfed condition (Table 3). Markhart (1985) stated that stomatal characteristics, such as stomatal density, aperture size, sensitivity change in internal and external water status. The diffusion resistance of the epidermis to water vapour transfer is a function of stomatal density, as well as pore size. In contrast, Hack (1974) also reported that stomatal pore or the sub stomatal cavity may change in relation to the moisture status of the plant.

The present findings also revealed that stomatal pore breadth decreased significantly under rainfed condition in both the surfaces than the irrigated condition (Table 4). Volkenburh and Davies (1977) reported similar results in cotton and soybean leaves. Singh (1978) reported that increase soil moisture improved leaf area and width of stomata of rye and wheat. In contrast, Ahmed (1994) reported that the size of stomata decreased significantly with the degree of water stress imposed to wheat seedling. The responses of stomata to leaf water status and environment are important in regulating transpiration and 
photosynthesis. Shimshi and Ephart (1975) reported that wheat cultivars differed widely in their stomatal aperture under adequate moisture conditions. Sullivan and Eastin (1975) pointed out that one might expect higher leaf temperatures when stomata are closed: thus the species that close their stomata earlier when stressed must have greater heat tolerance mechanisms. Glovar (1959) also reported changes in stomatal response after repeated stress periods. Sullivan and Eastin (1975) pointed out the necessity of considering previous growth conditions when evaluating stomatal response to water stress. From the results of the present study, it may be assumed that yield may be decreased with the decrease of stomatal aperture as well as stomatal pore breadth at rainfed condition.

\section{References}

Ahmed. S. 1994. Effect of water stress in wheat. Progress. Agric. 5(2):7-11.

Asana R. D., A. D. Saini and D. Ray. 1958. Studies in physiological analysis of yield. III. The rate of grain development in wheat in relation to photosynthetic surface and soil moisture. Plant Physiol. 11: 655-665.

Carr. D.J. and I. F. Wardlaw. 1965. The supply of photosynthetic assimilate to the grain from the grain from the leaf and ear of wheat. Aust J. Biol. Sc. 1:711 -719.

Glovar, J. 1959. Drought resistance in wheat. J. Agric. Sci. 153: 412-416.

Hack, H.R.B. 1974. The selection of an infiltration technique for estamating the degree of stomatal opening in leaves of field crops in the sudan and a discussion of the mechanism which controls the entry of test lequids. Ann. Bot. 55: 93-114.

Jones, H.G. 1977. Aspects of the water relations of spring wheat (Triticum aestivum L.) in response to induced drought. J. Agric. Sci. Camb. 88: 267- 282.

Kazemi, H., S.R. Chapman and F.H. McNeal. 1978. Variation in stornatal number in spring wheat cultivars. Cereal Res Comm. 6: 359-365.

Kriedemann P. 1966. The photosynthetic activity of the wheat ear. Ann. Bot. 30: 349363.

Kumer. A., S.D. Dhiman and S.K. Yadav. 1986. Stomatal frequency and distribution, water relations and micro-environment of wheat genotypes under irrigated and unirrigated conditions. Indian Journal of Ecology 13(2): 256 -265.

Markhart. A.H. 1985. Copparative water relation of Phaseolus vulgaris L. and Phaseolus acutifolius gray. Plant Physiol 77: 113-117.

Nayeem. K.A. and S.K. Garskin. 1990. Genetic variability, heritability and genetic advance of stomatal frequency in wbeat, triticale and barley. Indian J. of Genetics and Plant Breeding 50(2): 179-184.

Quinlan. J.D. and G.R. Sagar. 1965. Grain yield in two contrasting varieties of spring wheat. Ann. Bat. 29: 683-697.

Shimshi. D. and J. Ephrat. 1975. Stomatal behaviour of wheat cultivars in relation to their transpiration, photosynthesis and yield. Agron. J. 67: 326-331. 
Singh. D.P. 1978. Relation of soil moisture and air conditiomng irrigation to plant water balance, growth characteristics and nutrient uptake in rye and wheat. Biologia Plantarurn. 20(3): 16 1-163.

Sullivan, C.Y. and J. D. Eastin. 1975. In symposium on plant modification for more effective water use (J.E. Stone and J.E. Newman, eds.), Agric. Meteor. 14: 113-127.

Volkenburgh. E.V. and W.J. Davies. 1977. Leaf anatomy and water relations of plant grown in controlled environments and in the field. Crap Sci. 17: 353- 358.

Volden, H.D. and G.M. Simpson. 1967. Leaf area as an indicator of potential grain yield in wheat. Can. J. Plant. Sci. 47:359-365. 